\title{
Tearing mode analysis in tokamaks, revisited
}

\author{
Y. Nishimura, ${ }^{\text {a) }}$ J. D. Callen, and C. C. Hegna \\ Department of Engineering Physics, University of Wisconsin-Madison, Wisconsin 53706-1687
}

(Received 26 May 1998; accepted 8 September 1998)

\begin{abstract}
A new $\Delta^{\prime}$ shooting code has been developed to investigate tokamak plasma tearing mode stability in a cylinder and large aspect ratio $(\epsilon \leqslant 0.25)$ toroidal geometries, neglecting toroidal mode coupling. A different computational algorithm is used (shooting out from the singular surface instead of into it) to resolve the strong singularities at the mode rational surface, particularly in the presence of the finite pressure term. Numerical results compare favorably with Furth et al. [H. P. Furth et al., Phys. Fluids 16, 1054 (1973)] results. The effects of finite pressure, which are shown to decrease $\Delta^{\prime}$, are discussed. It is shown that the distortion of the flux surfaces by the Shafranov shift, which modifies the geometry metric elements, stabilizes the tearing mode significantly, even in a low- $\beta$ regime before the toroidal magnetic curvature effects come into play. (C) 1998 American Institute of Physics. [S1070-664X(98)02812-2]
\end{abstract}

\section{INTRODUCTION}

Understanding resistive magnetohydrodynamic (MHD) stability is important for long pulse tokamak operations, since tearing modes form magnetic islands, and if islands of incommensurate helicity overlap, they can induce plasma disruptions. ${ }^{1}$ Also, the existence of a single helicity magnetic island can deteriorate plasma confinement due to the change in magnetic field line topology. ${ }^{2,3}$ In resistive MHD, tearing mode stability is determined by a parameter delta prime $\left(\Delta^{\prime}\right)$, which was first defined by Furth et al..$^{4}$ a positive $\Delta^{\prime}$ implies instability. Recently, $\Delta^{\prime}$ was measured in the Tokamak Test Fusion Reactor (TFTR) supershot plasma experiments through an analysis of electron temperature fluctuations. ${ }^{5}$ It has been shown that when $m / n=2 / 1$ modes are present, $\Delta^{\prime}>0$. This can be explained by classical tearing mode theory. On the other hand, $\Delta^{\prime}$ is observed to be negative for cases with $m / n=3 / 2$ and $m / n=4 / 3$ modes, which indicates the presence of destabilizing neoclassical effects. ${ }^{6}$ The qualitative framework of tearing mode theory seems to be well established.

In toroidal tokamak plasmas, Fourier harmonics of tearing modes are correlated to each other, both through the poloidal mode coupling and nonlinear effects. These couplings can play an important role in destabilizing the modes on magnetic surfaces of incommensurate helicity. The final goal of our research is to investigate such a multimode coupled system, for example, as pursued in the PEST- 3 code. $^{7}$ However, the quantitative determination of the tearing mode stability parameter $\Delta^{\prime}$ still remains an essential issue: a precise prediction of stability becomes important, especially when the tearing modes are marginally stable $\left(\left|r_{s} \Delta^{\prime}\right| \lesssim 1\right.$, where $r_{s}$ is the mode rational surface radius). The value of $\Delta^{\prime}$ is sensitive to the local current gradient, even in the single helicity case. For the purpose of seeking optimized discharge current profiles for the experiments and simultaneous feedback control, ${ }^{8}$ it is important to understand and

${ }^{\text {a)} E l e c t r o n i c ~ m a i l: ~ n i s h i m y a @ j o v e . c o l o r a d o . e d u ~}$ clarify the nature of the numerical procedures that correctly relate $\Delta^{\prime}$ to the current profile.

A shooting-type code ${ }^{9}$ for determining the perturbed helical flux profile and hence $\Delta^{\prime}$ is compact, intuitively straightforward, and has fast convergence. Obtaining a $\Delta^{\prime}$ value for a single helicity case takes less than a second of CPU time on nominal workstation computers. This fast convergence is tractable for developing a feedback stabilization scheme for controlling tearing modes in tokamak discharges. ${ }^{8}$

Finite pressure effects were ignored in the previous analyses by Furth et al. ${ }^{9}$ and Wesson. ${ }^{10}$ This is due to the fractional power-like singularity that arises at the mode rational surface when pressure gradient effects are present. Furthermore, difficulties arise in separating the large and the small solutions near the rational surface. ${ }^{11}$ In this work, a different type of numerical algorithm was employed (integrated out from the singular surface rather than into it). The algorithm converges correctly in a high $\beta(\beta \geqslant 7 \%)$ regime for cylindrical geometry ( $\beta$ represents the ratio between the plasma and magnetic pressure). To check the validity of our computational results, we start (see Sec. III) by comparing our results with the Furth et al. ${ }^{9}$ results for zero pressure cases.

The effects of toroidal geometry are considered in this work. The effects can be separated into (1) distortion of the flux surfaces due to the Shafranov shift; (2) the existence of an averaged magnetic well; and (3) mode coupling effects due to the $1 / R$ dependence of the magnetic field, which is neglected here. We have included (1) and (2), and obtained $\Delta^{\prime}$ as a function of $\beta{ }^{12}$ The Shafranov shift appears as a global effect that enters through changes in the geometry metric elements. The second effect appears as a localized property near the mode rational surface; we have included toroidal curvature (the Mercier index ${ }^{13}$ ) in the analysis, but have not included resistive layer effects. ${ }^{14}$ The mode coupling effect can be neglected in the case of a rotating tokamak plasma, where shear flows are large and modes at different surfaces decouple. ${ }^{15}$ 
This paper is organized as follows. In Sec. II the original definition and the physical significance of $\Delta^{\prime}$ is discussed. In Sec. III the basic model of Furth et al. ${ }^{9}$ for the exterior region is presented and our $\Delta^{\prime}$ calculation is compared with their results. The logarithmic singularity at the resistive layer, as well as the algorithm for solving the boundary value problem, is discussed in detail. Finite pressure effects are discussed in Sec. IV. The effects of toroidal geometry are introduced in Sec. V. Finally, we summarize in Sec. VI.

\section{THE DEFINITION OF $\Delta^{\prime}$}

The physical significance of the sign of $\Delta^{\prime}$ can be understood intuitively from the one-dimensional magnetic field line diffusion equation given by Faraday's law and the Ohm's law: ${ }^{16}$

$$
\frac{\partial \widetilde{B}_{r}}{\partial t}=\frac{\eta}{\mu_{0}} \nabla^{2} \widetilde{B}_{r} \sim \frac{\eta}{\mu_{0}} \frac{\partial^{2} \widetilde{B}_{r}}{\partial r^{2}},
$$

where $\eta$ and $\mu_{0}$ stand for the plasma resistivity and vacuum magnetic permeability, respectively. According to Ref. 16, it can be seen that if $\partial^{2} \widetilde{B}_{r} / \partial r^{2}<0$, then $\partial \widetilde{B}_{r} / \partial t<0$ and the perturbation damps in the initial phase. On the other hand, if $\partial^{2} \widetilde{B}_{r} / \partial r^{2}>0$, then $\partial \widetilde{B}_{r} / \partial t>0$ and the perturbation grows.

The plasma column is divided into two "exterior regions" ${ }^{4}$ by a resistive layer of width $\delta$. The mode rational surface where $q=m / n$ resides in this thin layer. Eigenmode profiles for $\widetilde{B}_{r}=-i(m / r) \psi$ are connected from one exterior region to the other via resistive layer properties, where $\psi$ is the flux function. Thus, there are jump discontinuities in the slope between the two exterior regions. If the eigenfunction is locally convex (concave), the mode is stable (unstable). Furth $e t a l .^{4}$ defined $\Delta^{\prime}$ as the difference of the slopes for the flux function $\psi$ inside and outside the mode rational surface $x_{s}$ :

$$
\Delta^{\prime} \equiv \frac{\psi_{+}^{\prime}\left(x_{s}+\delta\right)-\psi_{-}^{\prime}\left(x_{s}-\delta\right)}{\psi\left(x_{s}\right)},
$$

where a positive $\Delta^{\prime}$ implies instability. Note that the $\Delta^{\prime}$ value indicates the relative jump in $\widetilde{B}_{r}$ across the inner layer, and hence corresponds to the existence of a current sheet in the inner layer.

\section{REVIEW OF CYLINDRICAL FORMULATION AND COMPUTATION}

In this section, the basic model equation (the exterior equation) and the results of Ref. 9 are reviewed. A cylindrical coordinate system is employed in the calculation, where $r$ is the minor radius, $\theta$ is the poloidal angle, and $z$ is in the toroidal direction. Combining the momentum balance equation, Faraday's law, Ohm's law, and plasma incompressibility in a cylindrical geometry, we obtain the Newcomb equation, ${ }^{9,17}$

$$
\frac{d^{2} \psi}{d r^{2}}+\frac{1}{H} \frac{d H}{d r} \frac{d \psi}{d r}-\frac{1}{H}\left[\frac{g}{F^{2}}+\frac{1}{F} \frac{d}{d r}\left(H \frac{d F}{d r}\right)\right] \psi=0,
$$

where

$$
\begin{aligned}
F \equiv \mathbf{k} \cdot \mathbf{B}=k B_{z}+(m / r) B_{\theta}=\frac{B_{z}}{R}(1-m / q), & \\
H \equiv & \frac{r^{3}}{k^{2} r^{2}+m^{2}}, \\
g \equiv & \frac{\left(m^{2}-1\right) r F^{2}}{k^{2} r^{2}+m^{2}}+\frac{k^{2} r^{2}}{k^{2} r^{2}+m^{2}} \\
& \times\left(r F^{2}+F \frac{2\left(k r B_{z}-m B_{\theta}\right)}{k^{2} r^{2}+m^{2}}+2 \mu_{0} \frac{d P}{d r}\right) .
\end{aligned}
$$

Note that the toroidal mode number is taken to be $n=1$ throughout this paper. After normalization using $x \equiv r / r_{s}\left(r_{s}\right.$ is the mode rational surface radius), $b \equiv B_{\theta} / B_{z}$, and $p$ $\equiv P / P_{0}=2 \mu_{0} P / B_{z}^{2} \beta \quad\left(\beta \equiv 2 \mu_{0} P_{0} / B_{z}^{2}\right.$ is a figure of merit that represents the ratio between the plasma and magnetic pressure, and $P_{0}$ is the peak pressure), Eq. (3) reduces to

$$
\psi^{\prime \prime}+g_{2}(x) \psi^{\prime}-g_{1}(x) \psi=0,
$$

where we have further defined

$$
\begin{aligned}
& g_{1}(x) \equiv \frac{1}{H}\left(\frac{g}{F^{2}}+\frac{1}{F}\left(H F^{\prime}\right)^{\prime}\right), \\
& g_{2}(x) \equiv \frac{H^{\prime}}{H} .
\end{aligned}
$$

In Eq. (4), the prime denotes differentiation with respect to $x$. Here, the poloidal magnetic field profile, and safety factor are taken to be the peaked profile of Ref. 9:

$$
\begin{aligned}
& b(x)=\frac{x}{\left(1+x^{2}\right)}, \\
& q(x)=q_{0}\left(1+x^{2}\right) .
\end{aligned}
$$

The plasma boundary is located at $x_{b}=2$, while the mode rational surface is varied by changing the $q_{0}$ value. The pressure profile was taken to be $p=0$ for the zero $\beta$ case and $p(x)=1-\left(x / x_{b}\right)^{2}$ for the finite $\beta$ case, which we discuss in Sec. IV.

The numerical algorithm of the shooting method will now be explained. In the vicinity of the rational surface and in the absence of plasma pressure $(\beta=0)$, Eq. (4) reduces to the form

$$
\frac{d^{2} \psi}{d \mathscr{C}^{2}}-\frac{\kappa}{\mathscr{C}} \psi=0,
$$

where $\mathscr{C}=x-x_{s}$ and $\kappa=g_{1}\left(x_{s}\right)$. Two inner limits of the exterior asymptotic solutions for $\psi \equiv \psi_{\mathrm{I}}$ or $\psi_{\mathrm{III}}$,

$$
\begin{aligned}
\psi= & \left(1+\kappa \mathscr{C} \ln |\mathscr{C}|+\frac{1}{2} \kappa^{2} \cdot \mathscr{C}^{2} \ln |\mathscr{X}|-\frac{3}{4} \kappa^{2} \mathscr{C}^{2}+\cdots\right) \\
& +A_{\mathrm{I}, \mathrm{III}}\left(\mathscr{C}+\frac{1}{2} \kappa \mathscr{C}^{2}+\frac{1}{12} \kappa^{2} \mathscr{X}^{3}+\cdots\right),
\end{aligned}
$$


are matched to the numerical solutions at $\mathscr{B}= \pm \delta$. Here, the subscripts "I" and "III" denote the two exterior regions inside and outside the mode rational surface $x_{s}$. We solve Eq. (4) by numerically shooting away from the singular surface with an arbitrary boundary condition toward the other ends at $x=0$ and $x=x_{b}$, and iterate the constants $A_{\mathrm{I}}$ and $A_{\mathrm{III}}$ until the boundary conditions there are satisfied. (This is in the opposite way from Furth et al., ${ }^{9}$ where the shooting was done from the boundaries inward and numerical solutions near $x_{s}$ were fit to the asymptotic solution.) We solve Eq. (4) in the two regions $0 \leqslant x \leqslant x_{s}$ and $x_{s} \leqslant x \leqslant x_{b}$, obtaining $\psi_{\mathrm{I}}$ and $\psi_{\mathrm{III}}$. Defining $\mathbf{Y}=\left(y_{1}, y_{2}, y_{3}, y_{4}\right)=\left(\psi, \psi^{\prime}, \partial \psi / \partial A_{\mathrm{I}, \mathrm{III}}\right.$, $\left.\partial \psi^{\prime} / \partial A_{\text {I,III }}\right)$, Eq. (4) reduces to a system of simultaneous ordinary differential equations,

$$
\frac{d \mathbf{Y}}{d x}=\left(\begin{array}{c}
y_{1}^{\prime} \\
y_{2}^{\prime} \\
y_{3}^{\prime} \\
y_{4}^{\prime}
\end{array}\right)=\left(\begin{array}{c}
y_{2} \\
-g_{2}(x) y_{2}+g_{1}(x) y_{1} \\
y_{4} \\
-g_{2}(x) y_{2}+g_{1}(x) y_{1}
\end{array}\right),
$$

with boundary conditions at $x=x_{s} \pm \delta$ :

$$
\left(\begin{array}{l}
y_{1} \\
y_{2} \\
y_{3} \\
y_{4}
\end{array}\right)=\left(\begin{array}{c}
\left(1+\kappa \delta \ln |\delta|+\frac{1}{2} \kappa^{2} \delta^{2} \ln |\delta|-\frac{3}{4} \kappa^{2} \delta^{2}\right)+A_{\mathrm{I}, \mathrm{III}}\left(\delta+\frac{1}{2} \kappa \delta^{2}+\frac{1}{12} \kappa^{2} \delta^{3}\right) \\
\kappa(\ln \delta+1)+\kappa^{2}(\delta \ln |\delta|-\delta)+A_{\mathrm{I}, \mathrm{III}}\left(1+\kappa \delta+\frac{1}{4} \kappa^{2} \delta^{2}\right) \\
\delta+\frac{1}{2} \kappa \delta^{2}+\frac{1}{12} \kappa^{2} \delta^{3} \\
1+\kappa \delta+\frac{1}{4} \kappa^{2} \delta^{2}
\end{array}\right)
$$

A fourth-order Runge-Kutta-Gill method ${ }^{18}$ is used for the numerical integration. One changes the unknown constant $A_{\text {I,III }}$ until the values $A^{l+1}-A^{l}=-(\psi) /(\partial \psi / \partial A)$ at the boundaries converge ( $l$ represents the iteration step). The value of $\Delta^{\prime}$ is calculated by

$$
\Delta^{\prime}=\frac{\psi_{\mathrm{III}}^{\prime}\left(x_{s}+\delta\right)-\psi_{\mathrm{I}}^{\prime}\left(x_{s}-\delta\right)}{\psi\left(x_{s}\right)}=A_{\mathrm{III}}-A_{\mathrm{I}} .
$$

For a comparison with Furth et al., ${ }^{9}$ we take the poloidal/toroidal mode numbers to be $m / n=2 / 1$, while a large aspect ratio is represented by $k r_{0}=0.05$. Figure 1(a) shows the eigenmode profile, while Fig. 1(b) shows the value of $\Delta^{\prime}$ as a function of mode rational surfaces $x_{s}$. Figure 1(b) implies that the tearing modes are relatively stable when the mode rational surfaces are closer to the plasma boundary. The results match exactly with Fig. 1 of Ref. 9. (Note that the numerical algorithm we employed is quite different.) We have also reproduced the numerical results by Wesson ${ }^{10}$ (see the Appendix).

\section{EIGENMODE SOLUTIONS IN THE PRESENCE OF FINITE PRESSURE}

An algorithm for cases with the pressure term is discussed in this section. In the presence of finite pressure effects, the exterior equation, Eq. (4), has strong singularities with two independent solutions called the large (dominant) and the small (subdominant), ${ }^{11}$ exhibiting different fractional power-like asymptotic behaviors:

$$
\psi=A_{\mathrm{I}}|\mathscr{B}|^{h+1}-B_{\mathrm{I}}|\mathscr{B}|^{-h},
$$

in region $\mathrm{I}$, and

$$
\psi=A_{\mathrm{III}}|\mathscr{B}|^{h+1}+B_{\mathrm{III}}|\mathscr{B}|^{-h},
$$

in region III. Here $h=-\frac{1}{2}+\frac{1}{2} \sqrt{1-4 D_{s}}, \quad D_{s} \equiv \beta$ $\left.\left(-2 q^{2} / q^{\prime 2} B_{z}^{2} x\right)(d p / d x)\right|_{x=x_{s}},{ }^{11}$ and $\mathscr{C}=x-x_{s}$, as before. Note that in the $h=0$ limit, this reduces to the same form as the zero pressure case. As in the $\beta=0$ case, the asymptotic solutions Eqs. (12) and (13) at the resonant surface are matched to the numerical solution.

Defining $\quad \mathbf{Y}=\left(y_{1}, y_{2}, y_{3}, y_{4}, y_{5}, y_{6}\right)=\left(\psi, \psi^{\prime}, \partial \psi / \partial A\right.$, $\left.\partial \psi^{\prime} / \partial A, \partial \psi / \partial B, \partial \psi^{\prime} / \partial B\right)$,

$$
\frac{d \mathbf{Y}}{d x}=\left(\begin{array}{c}
y_{1}^{\prime} \\
y_{2}^{\prime} \\
y_{3}^{\prime} \\
y_{4}^{\prime} \\
y_{5}^{\prime} \\
y_{6}^{\prime}
\end{array}\right)=\left(\begin{array}{c}
y_{2} \\
-y_{2} g_{2}(x)+g_{1}(x) y_{1} \\
y_{4} \\
-y_{4} g_{2}(x)+g_{1}(x) y_{3} \\
y_{6} \\
-y_{6} g_{2}(x)+g_{1}(x) y_{5}
\end{array}\right),
$$

we shoot with a boundary condition at $x=x_{s} \pm \delta$,

$$
\left(\begin{array}{c}
y_{1} \\
y_{2} \\
y_{3} \\
y_{4} \\
y_{5} \\
y_{6}
\end{array}\right)=\left(\begin{array}{c}
A|\delta|^{h+1} \pm B|\delta|^{-h} \\
A(h+1)|\delta|^{h} \pm B h|\delta|^{-h-1} \\
|\delta|^{h+1} \\
(h+1)|\delta|^{h} \\
\pm|\delta|^{-h} \\
\pm h|\delta|^{-h-1}
\end{array}\right)
$$

One changes the unknown constants $A$ and $B$ until

$$
\begin{aligned}
\left(\begin{array}{l}
A^{(l+1)}-A^{(l)} \\
B^{(l+1)}-B^{(l)}
\end{array}\right)= & \left(\begin{array}{ll}
\partial y_{1} / \partial A & \partial y_{1} / \partial B \\
\partial y_{2} / \partial A & \partial y_{2} / \partial B
\end{array}\right)^{-1} \\
& \times\left(\begin{array}{ll}
y_{1}-y_{1}(x=0 & \text { or } \left.x=x_{b}\right) \\
y_{2}-y_{2}(x=0 & \text { or } \left.x=x_{b}\right)
\end{array}\right),
\end{aligned}
$$

converges at $x=0$ and $x=x_{b}$. The value of $\Delta^{\prime}$ is calculated by

$$
\Delta^{\prime} \equiv \frac{A_{\mathrm{III}}}{B_{\mathrm{III}}}-\frac{A_{\mathrm{I}}}{B_{\mathrm{I}}} .
$$


(a)

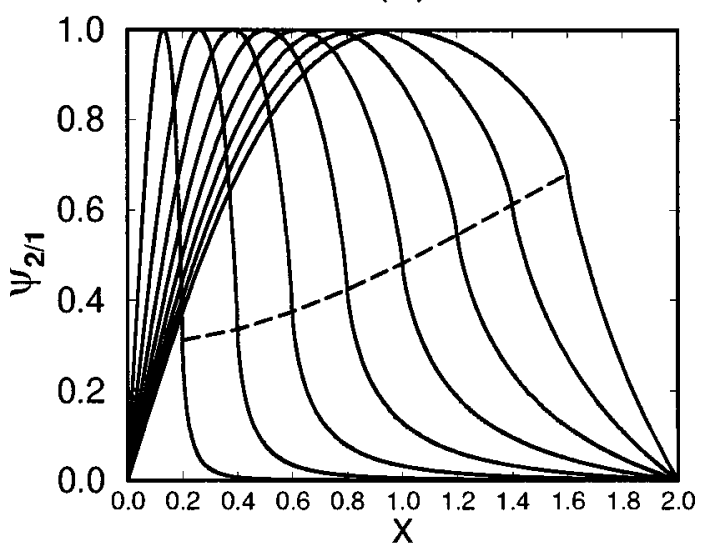

(b)

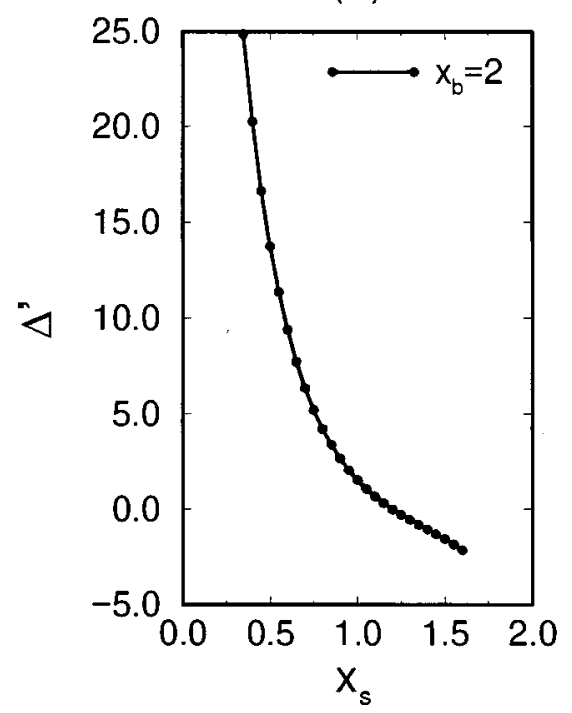

FIG. 1. (a) The eigenmode profile for an $m / n=2 / 1$ mode. The position of the mode rational surface $x_{s}$, indicated by dashed lines, is varied. These results correspond to Fig. 2 of Ref. 9. (b) Values of $\Delta^{\prime}$ as a function of $x_{s}$, which corresponds to the $m=2, x_{b}=2$ case of Fig. 1 of Ref. 9 .

Since we have two unknowns $A$ and $B$ at $x=x_{s} \pm \delta$, we need additional boundary conditions at $x=0$ and $x=x_{b}$ for $\psi^{\prime}$. However, the value of $\Delta^{\prime}$ is independent of these additional boundary conditions, since only the ratio $A / B$ in each region I and III is required to obtain $\Delta^{\prime}$. Note that by setting $B$ $=1$ and $h=0$, the system of Eqs. (14) and (15) reduces to the form of Eqs. (9) and (10).

Figure 2(a) shows the resultant eigenfunction of an $m / n=2 / 1$ mode with $\beta=7 \%(h=-0.076)$, which has singular behavior in the vicinity of the mode rational surface. Here, $x_{s}=1$ and $x_{b}=2$ were taken. Figure 2(b) expands the singular behavior in the vicinity of $x_{s}$; the analytical solutions (solid line) given by Eqs. (12) and (13) are successfully matched to the numerical solutions (dashed line). Figure 2(c) shows the value of $\Delta^{\prime}$ vs $\beta$ with the layer width set to $\delta$ $=10^{-7}$. The effect of the finite pressure term decreases the $\Delta^{\prime}$ value, and thus stabilizes the tearing mode, even in a cylindrical tokamak. Note that $D_{s} \geqslant 0(h \leqslant 0)$ in a cylinder, (a)

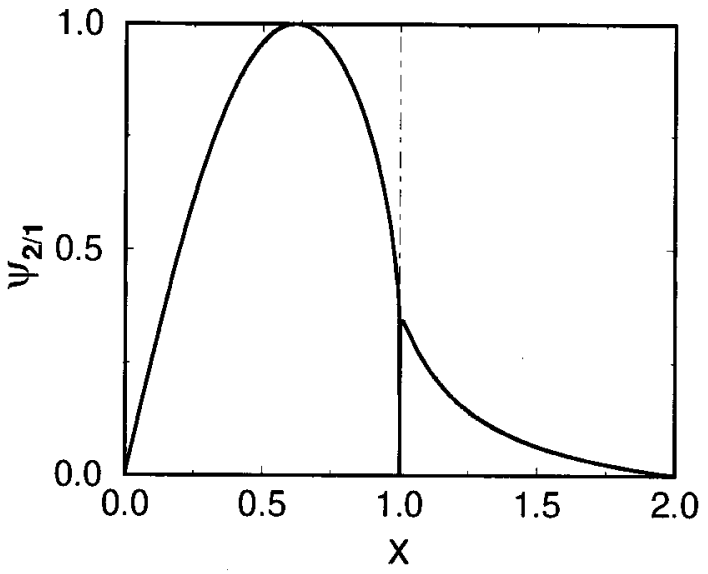

(b)

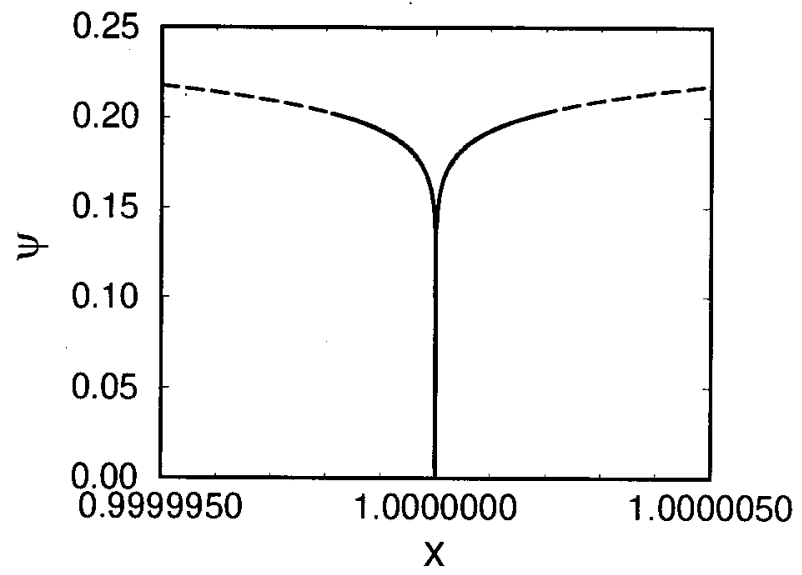

(c)

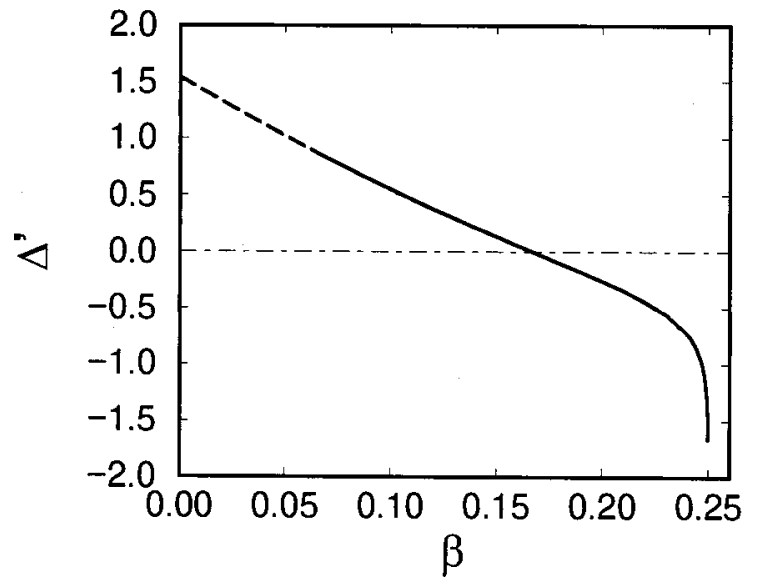

FIG. 2. (a) The eigenmode profile for an $m / n=2 / 1$ mode. (b) Expansion of the eigenfunction behavior in the vicinity of $x_{s}$. Both analytical solutions (solid line) and the numerical solutions (dashed line) are shown. (c) Here $\Delta^{\prime}$ vs $\beta$ in the high $\beta(\beta \geqslant 7 \%)$ regime. The dashed line signifies regions where layer widths wider than physical ones $\left(\delta \sim e^{1 / h}\right)$ are imposed.

while $D_{s} \leqslant 0 \quad(h \geqslant 0)$ for a $q>1$ surface in a toroidal geometry. ${ }^{14}$

In a $\beta \rightarrow 0$ limit, the $\Delta^{\prime}$ value reduces to the zero pressure case $\Delta^{\prime}=1.54$ [see Fig. 1(b)]. In this $\beta \rightarrow 0$ limit (but with the pressure term), the second terms in Eqs. (12) and 
TABLE I. The relation between $\beta, h$, and $\delta$. Here, $D_{s}=\beta$ with a peaked profile.

\begin{tabular}{ccc}
\hline \hline$\beta(\%)$ & $h$ & Layer width $\delta$ \\
\hline 1 & -0.010 & $3.7 \times 10^{-44}$ \\
5 & -0.053 & $6.3 \times 10^{-9}$ \\
7 & -0.076 & $1.9 \times 10^{-6}$ \\
10 & -0.11 & $1.1 \times 10^{-4}$ \\
20 & -0.27 & $2.1 \times 10^{-2}$ \\
\hline \hline
\end{tabular}

(13) become constants and reduce to the form of Eq. (8). In Fig. 2(c), the $\Delta^{\prime}$ value in a relatively high $\beta$ regime $(\beta$ $\geqslant 7 \%$ ) is calculated correctly. (See Table I for the estimated scale length $\delta$ as function of $\beta$.) Note that the value of $\Delta^{\prime}$ approaches -2 as $D_{s}$ approaches $\frac{1}{4}$, where the large and the small solutions become comparable. This feature can be predicted analytically from Eq. (17); $A_{\mathrm{III}} / B_{\mathrm{III}}-A_{\mathrm{I}} / B_{\mathrm{I}}=-1-1$ $=-2$. These results guarantee that the algorithm is mathematically appropriate.

When $\delta \leqslant 10^{-8}$, the numerical method becomes troublesome. The limit of the numerical method depends on the ratio of the coefficients $\partial_{A} y_{1,2}$ and $\partial_{B} y_{1,2}$ in Eq. (16). For extremely small layer width $\delta$, numerical truncations in calculating the right side of Eq. (16) obscure the mathematical separation between the large and the small solutions.

In a $\beta \rightarrow 0$ limit, the characteristic layer scale width for pressure effect $\delta \sim e^{1 / h}$ becomes literally infinitesimal (for example, $\delta \sim 10^{-44}$ for $\beta=1 \%$ and $\delta \sim 10^{-6}$ for $\beta=7 \%$; see Table I). However, needless to say, at the same time, the pressure effect becomes negligible. Furthermore, in the range $0 \% \leqslant \beta \leqslant 7 \%$, one can conjecture that $\Delta^{\prime}$ takes on values that smoothly match with $\Delta^{\prime}=1.54$ at $\beta=0$, and the curve of Fig. 2(c) in the $\beta \geqslant 7 \%$ regime. Physically, a layer must have a finite width: a layer width $\delta$ smaller than the ion Larmor radius (on the order of $10^{-5}$, even in a plasma of a temperature as low as $100 \mathrm{eV}$ ) is only a metaphysical consideration. In short, we see that the shooting method presented here extracts the finite pressure effects on $\Delta^{\prime}$, within a reasonable parametric regime for tokamak plasmas. Finite pressure effects in toroidal geometry are discussed in the next section.

\section{EIGENMODE IN A TOROIDAL GEOMETRY}

In a toroidal equilibrium, straight magnetic field lines ${ }^{19}$ are represented by

$$
B=I(\rho) \nabla \zeta+\nabla \zeta \times \nabla \psi_{\mathrm{eq}}(\rho)=\nabla \psi_{\mathrm{eq}}(\rho) \times \nabla[q(\rho) \theta-\zeta],
$$

where $\zeta$ is the toroidal angle and $\rho$ is the equilibrium magnetic surface label. With a relation $\partial \psi_{\mathrm{eq}} / \partial \rho=I \rho / q$, the fluxsurface-averaged exterior equation in toroidal geometry ${ }^{12}$ reduces to (small aspect ratio $\epsilon \ll 1$ and $I=$ const, assumed for simplicity)

$$
\begin{gathered}
\frac{1}{x} \frac{\partial}{\partial x} x\left\langle g^{\rho \rho}\right\rangle \frac{\partial \psi}{\partial x}-\frac{m^{2}}{x^{2}}\left\langle g^{\theta \theta}\right\rangle \psi-\frac{m q}{x(m-n q)} \frac{d j}{d x} \psi \\
-\beta \frac{n^{2} q^{2}}{x^{2}(m-n q)^{2}} \frac{d p}{d x} \frac{d\left\langle R^{2}\right\rangle}{d x} \psi=0,
\end{gathered}
$$

where $\theta$ is the poloidal angle, $j(x)$ stands for the toroidal current, and $R=R(\rho, \theta)$ stands for the major radius. Here, \langle\rangle (a)

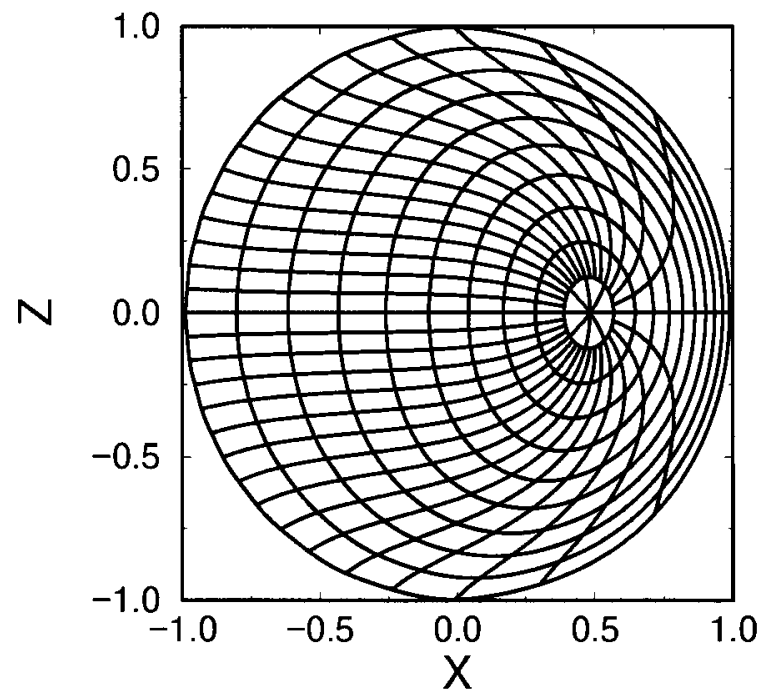

(b)

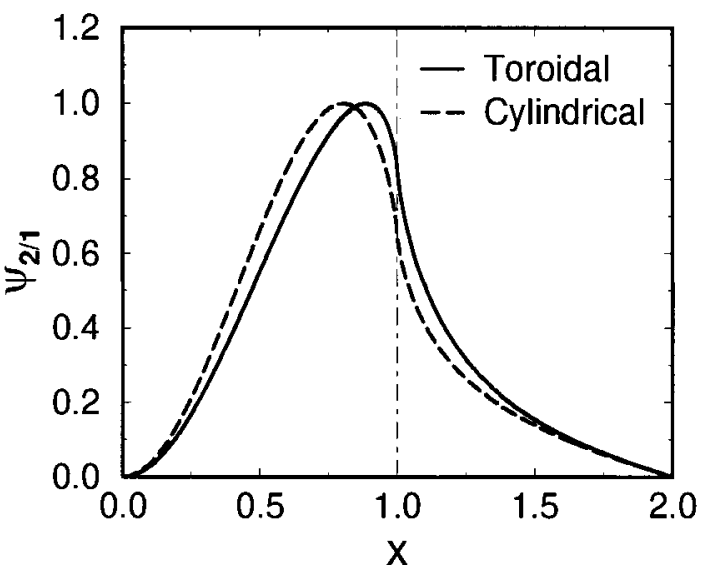

FIG. 3. (a) The equilibrium flux surfaces and $\theta$ contour lines in a toroidal geometry with $\beta=10 \%$, which show geometrical distortions. (b) The eigenmode profile of $\psi_{2 / 1}$. The solid line is for a toroidal geometry with $\beta$ $=10 \%$ and the dashed line is for a cylindrical limit (the pressure gradient drive term in the eigenmode equation is absent for both lines).

stands for a flux surface average. The magnetic well ${ }^{20}$ is approximated by $(-x)\left(d\left\langle R^{2}\right\rangle / d x\right)$; as stated in Ref. 12, a more careful derivation of the pressure term yields the " $\mathrm{E}+\mathrm{F}+\mathrm{H}$ ', terms of Glasser, Greene, and Johnson. ${ }^{14}$ Note that in a cylindrical plasma with a monotonically decreasing current, $d\left\langle R^{2}\right\rangle / d x \geqslant 0$, while $d\left\langle R^{2}\right\rangle / d x \leqslant 0$ can occur in a torus, since a good curvature region (a magnetic well) exists in a tokamak for $q>1 .^{21}$

From a mathematical viewpoint, this second-order ordinary differential equation gives a system of first-order equations that are similar to the cylindrical ones, except for the radially dependent functions $\left\langle g^{\rho \rho}\right\rangle,\left\langle g^{\theta \theta}\right\rangle$ (metric elements), and $\left\langle R^{2}\right\rangle$. The toroidicity effects are included in these latter functions. In this study, radial profiles of the flux-surfaceaveraged metric elements are obtained from an equilibrium code RSTEQ. ${ }^{22}$ Figure 3(a) exhibits the grids of the straight field line, PEST $^{19}$ coordinate system-both the equilibrium flux contours and the $\theta$ contours at $\beta=10 \%$. In Fig. 3(a), the abscissa $X=(R-1) / \epsilon$ and the ordinate $Z$ are horizontal and 
vertical dimensionless minor radius coordinates, respectively. In Fig. 3(a), the area of each grids represents the volume element $\boldsymbol{\nabla} \rho \times \boldsymbol{\nabla} \theta \cdot \boldsymbol{\nabla} \zeta=R^{-2}(\rho, \theta)$. Note that the equation reduces to the cylindrical version of the Newcomb equation ${ }^{10}$ (see the Appendix) in the $\left\langle g^{\rho \rho}\right\rangle=\left\langle g^{\theta \theta}\right\rangle=1$ limit.

The dashed line in Fig. 3(b) is the eigenmode profile in a toroidal geometry with $\beta=10 \%$, while the solid line is the profile in the cylindrical limit. [In Fig. 3(b), $\beta$ has been taken to be zero in Eqs. (3) and (19) to bring out the Shafranov shift, geometry effects.] The safety factor was taken to be $q(x)=1+x^{2}$, so that the $m / n=2 / 1$ mode rational surface is located at $x_{s}=1.0$ (the plasma boundary located at $x_{b}=2.0$ ). The dashed line (S-Shafranov shift) in Fig. 4(a) shows the value of $\Delta^{\prime}$ as function of $\beta$ [see Table II for the corresponding Shafranov shift $\Delta_{s}$; a magnetic axis shift measured in the $(X, Z)$ coordinate system]. The distortion of the flux surfaces (Shafranov shift effects) from the first and the second term of Eq. (19) significantly contribute to the decrease of $\Delta^{\prime}$ with $\beta$.

The curvature effect ${ }^{13}$ is now investigated quantitatively by including the $\beta \neq 0$ curvature term in Eq. (19). As a reminder, in toroidal geometry the $D_{s}$ value of Eq. (13) is replaced by ${ }^{14}$

$$
\begin{aligned}
D_{s} & \left.\equiv \beta\left(\frac{-q^{2}}{q^{\prime 2} x^{2}}\right)\left(\frac{d p}{d x}\right)\left(\frac{1}{\left\langle g^{\rho \rho}\right\rangle} \frac{d\left\langle R^{2}\right\rangle}{d x}\right)\right|_{x=x_{s}} \\
& \left.\simeq \beta\left(\frac{-2 q^{2}}{q^{\prime 2} x}\right)\left(\frac{d p}{d x}\right)\left(1-q^{2}\right)\right|_{x=x_{s}} .
\end{aligned}
$$

The last relation is given in the limit of a large aspect ratio. ${ }^{21}$ [The first form of Eq. (20) is employed for computation.] Thus, the $D_{s}$ value in a toroidal system can be negative ( $h$ can be positive) when $q$ is above unity. If $h$ is positive, the asymptotic solution given by Eqs. (12) and (13) gives rise to a $1 / \mathscr{C}^{h}$-like singularity $(|\psi| \rightarrow \infty$ at $\mathscr{X} \rightarrow 0)$. In contrast to the $h \leqslant 0$ case discussed in Sec. IV, the numerical truncation in Eq. (16) will be severe in higher $\beta$ cases rather than for lower $\beta$ cases because of the change in the sign of $D_{s}$ and thus the power $h$. Figure 4(b) shows an eigenfunction of an $m / n=2 / 1$ mode in a torus with $\beta=7 \%$, which has a positive spike in the vicinity of the mode rational surface. This is expected analytically from Eqs. (12) and (13). Figure 4(c) shows the detail of the singular behavior in the vicinity of $x_{s}$; the analytical solutions (solid line), Eqs. (12) and (13), are successfully matched to the numerical solutions (dashed line).

The dot-dashed line in Fig. 4(a) shows the value of $\Delta^{\prime}$ as a function of $\beta$ in the presence of the pressure term $(\mathrm{C}-$ curvature effect), but without toroidal shape effects (the metric elements are set to their cylindrical limits). The solid line in Fig. 4(a) shows the value of $\Delta^{\prime}$ in the presence of both of the effects. As one can see from the difference of the three lines in Fig. 4(a), toroidal geometry effects (S) stabilize the tearing mode significantly, even in a low $\beta$ regime before the curvature effect (C) comes into play, for the typical tokamak $q$ profile and a pressure profile we have employed (see Fig. 5 ). More generally, the curvature effect is expected to be modest, unless one enters an extremely high $\beta$ regime, or extremely low shear region where the $1 / q^{\prime 2}$ in $D_{s}$ becomes (a)

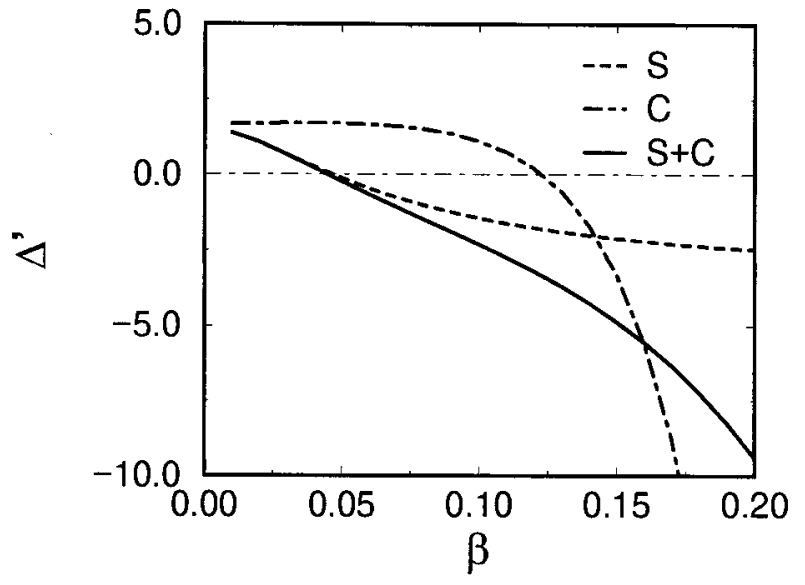

(b)

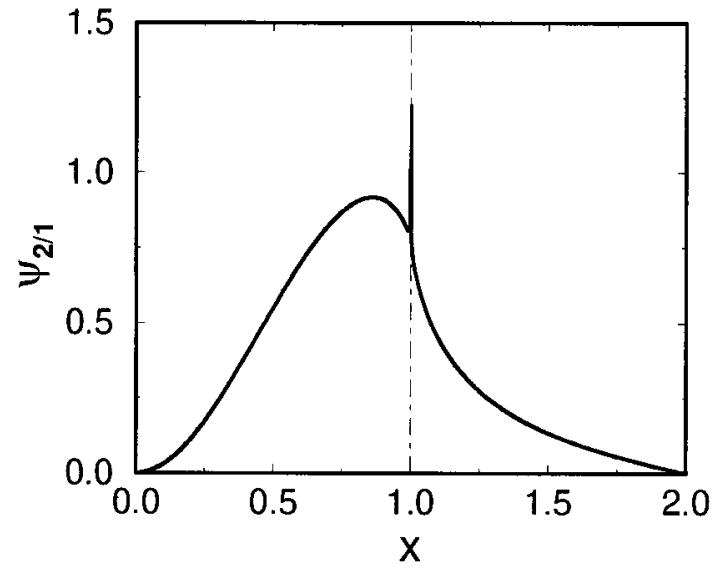

(c)

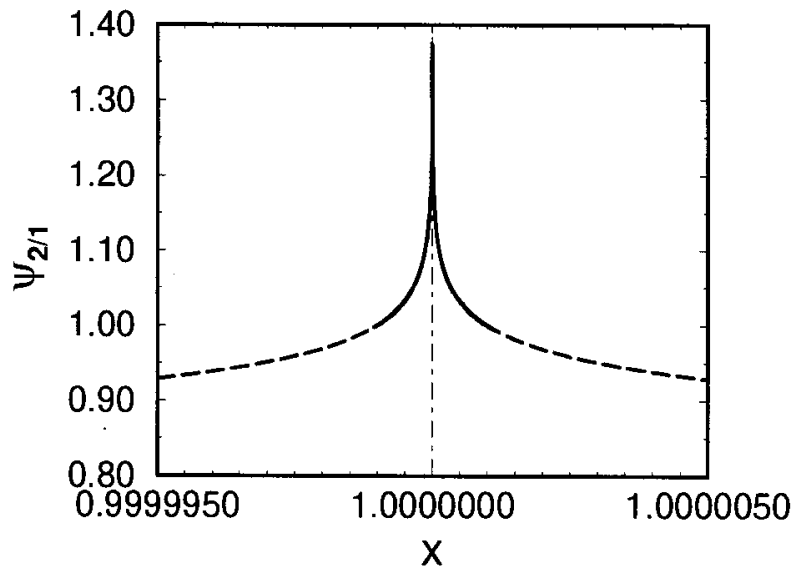

FIG. 4. (a) We see $\Delta^{\prime}$ as function of $\beta$ [S (Shafranov) indicates in the absence of the pressure gradient drive term, but with toroidal shape, $\mathrm{C}$ (curvature) in the presence of the pressure gradient drive term, but without toroidal geometrical effects $\left(\left\langle g^{\rho \rho}\right\rangle=\left\langle g^{\theta \theta}\right\rangle=1\right)$, and $\mathrm{S}+\mathrm{C}$ both the toroidal effects and pressure-drive effects present]. (b) The eigenmode profile of an $m / n=2 / 1$ mode with $\beta=7 \%$. (c) An expanded eigenfunction in the vicinity of $x_{s}$. Analytical solutions (solid line) and the numerical solutions (dashed line) are both shown.

influential. The line (C) in Fig. 4(a) supports this latter fact numerically. Note that the curvature effect is rather a localized layer property, while the Shafranov shift is a global effect obtained via the metric elements. 
TABLE II. The relation between $\beta$ and Shafranov shift $\Delta_{S}$ in the equilibrium with $q(x)=1+x^{2}$.

\begin{tabular}{cc}
\hline \hline$\beta(\%)$ & $\Delta_{s}$ \\
\hline 1 & 0.14 \\
2 & 0.21 \\
3 & 0.27 \\
5 & 0.36 \\
10 & 0.48 \\
20 & 0.60 \\
\hline
\end{tabular}

\section{SUMMARY AND DISCUSSION}

In this paper, we have reviewed tearing mode theory and computations in a cylinder and extended the studies to axisymmetric toroidal geometries. A new numerical algorithm that shoots out from the singular surface rather than in toward the singular layer was developed and employed. Numerical results obtained from the shooting code for cylindrical cases matched results obtained previously by Furth et al. ${ }^{9}$

The effect of finite pressure on tearing modes has been discussed. For the first time, the $\Delta^{\prime}$ value as a function of $D_{s}$ has been reported. It has been shown that, with the new algorithm we employed (shooting away from the mode rational surface rather than into it), shooting methods resolve the singularities $^{9}$ due to the finite pressure effect. The method guarantees separation of the large and the small solutions in the vicinity of a resonant surface. It has been demonstrated that the method is convergent for a relatively high $\beta$ regime $(\beta \geqslant 7 \%)$ for cylinders and low $\beta$ regime $(\beta \lesssim 20 \%)$ for toroidal geometries. It has been shown that the finite pressure effects stabilize the constant- $\psi$ tearing mode in a high $\beta$ ( $\beta$ $\geqslant 7 \%$ ) cylindrical geometry.

In toroidal geometries, it is suggested that the distortion of the flux surfaces by large Shafranov shifts (a macroscopic effect) stabilizes the tearing mode. The magnetic well effect ${ }^{13}$ was investigated quantitatively, as well. By comparing the change in $\Delta^{\prime}$ as a function of $\beta$, it has been shown that the distortion of the flux surface (or Shafranov shift) stabilizes the tearing mode significantly, even in a low $\beta$ regime (near the $\beta$ limit), before the magnetic well effect

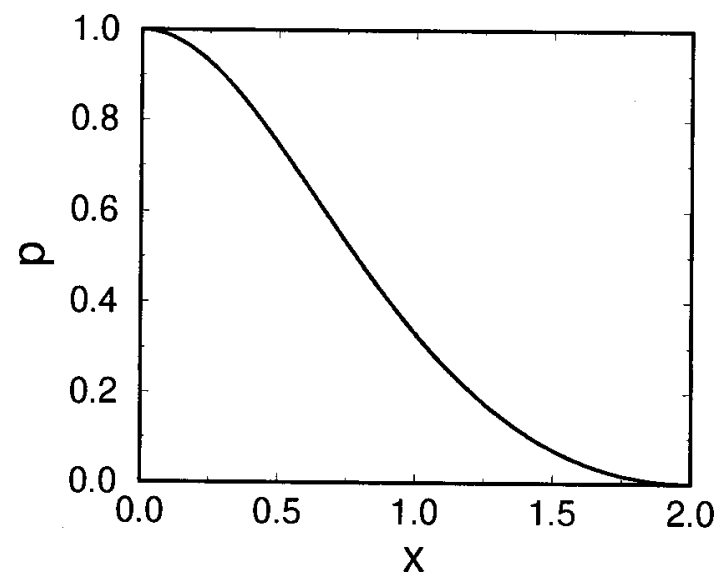

FIG. 5. The Grad-Shafranov equilibrium pressure profile employed for Figs. 4(a), (b), and (c). comes into play. Thus we believe that, for practical purposes in estimating $\Delta^{\prime}$ in the linear stage, the pressure term can be neglected unless one enters an extremely high $\beta$ regime, or an extremely low shear region. Inclusion of the pressure gradient term merely complicates the prediction of tearing mode stability.

The analysis of mode coupling of incommensurate helicity perturbations is beyond the scope of this paper and will be a subject for future work.

\section{ACKNOWLEDGMENTS}

One of the authors (Y.N.) is grateful to Dr. C. Ren and Dr. F. L. Waelbroeck for their valuable suggestions. Y. N. also thanks Dr. V. E. Lynch and Dr. J-N. G. Leboeuf for kind assistance in the usage of the equilibrium code RSTEQ.

This research was supported by United States Department of Energy Grant No. DE-FG02-86ER53218.

\section{APPENDIX: REVIEW OF WESSON'S ANALYSIS AND COMPUTATION}

In this Appendix, the results of Ref. 10 will be reviewed. In the large aspect ratio limit, the Newcomb equation, Eq. (4), reduces to

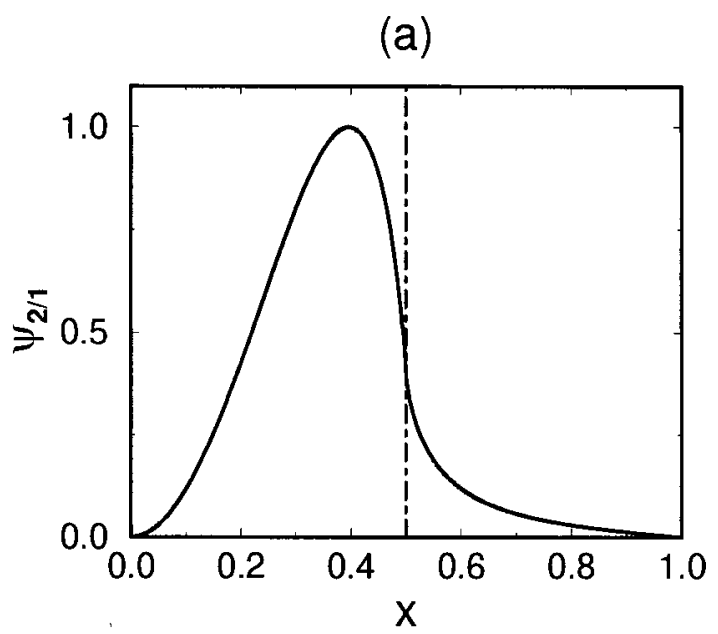

(b)

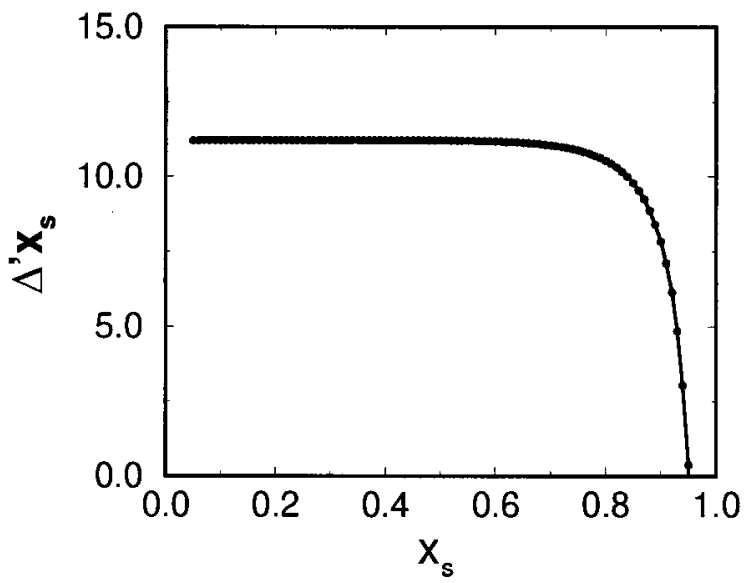

FIG. 6. (a) The eigenmode profile for an $m / n=2 / 1$ mode. (b) Here $\Delta^{\prime}$ vs $x_{s}$. These results correspond to Fig. 6.7.1 of Ref. 10. 


$$
\psi^{\prime \prime}+\frac{1}{x} \psi^{\prime}-g(x) \psi=0,
$$

where

$$
g(x)=\frac{m^{2}}{x^{2}}+\frac{m q}{x(m-n q)} \frac{d j}{d x}+2 \beta \frac{n^{2} q^{2}}{x(m-n q)^{2}} \frac{d p}{d x} .
$$

Wesson ${ }^{10}$ used current profiles in the form of $j(x)=(1$ $\left.-x^{2}\right)$, and correspondingly $b(x)=x / 2-x^{3} / 4$ and $q(x)$ $=4 \epsilon /\left(2-x^{2}\right)$. Here $\epsilon=\left(2-x_{s}^{2}\right)\left(q_{m / n} / 4\right)$ stands for the inverse aspect ratio. The numerical algorithm applied for Eq. (A1) is the same as in Sec. III. The value of $\Delta^{\prime}$ is calculated by $\Delta^{\prime}=A_{\mathrm{II}}-A_{\mathrm{I}}$, as before. Figure 6(a) shows the eigenmode profile for an $m / n=2 / 1$ mode with a mode rational surface located at $x_{s}=0.5$. Figure 6(b) shows the value of $\Delta^{\prime}$ as a function of mode rational surfaces $x_{s}$. The results match with Fig. 6.7.1 of Ref. 10.

${ }^{1}$ P. H. Diamond, R. D. Hazeltine, Z. G. An, B. A. Carreras, and H. R. Hicks, Phys. Fluids 27, 1449 (1984); S. Tsuji, Y. Nagayama, K. Miyamoto, K. Kawahata, N. Noda, and S. Tanahashi, Nucl. Fusion 25, 305 (1985).

${ }^{2}$ J. D. Callen, B. V. Waddell, B. Carreras, M. Azumi, P. J. Catto, H. R. Hicks, J. A. Holmes, D. K. Lee, S. J. Lynch, J. Smith, M. Soler, K. T. Tsang, and J. C. Whitson, in Plasma Physics and Controlled Nuclear Fusion Research 1978, Proceedings of the 7th International Conference, Innsbruck (International Atomic Energy Agency, Vienna, 1979), Vol. 1, p. 415 .

${ }^{3}$ Y. Nishimura and M. Azumi, Phys. Plasmas 4, 2365 (1997).

${ }^{4}$ H. P. Furth, J. Killeen, and M. N. Rosenbluth, Phys. Fluids 6, 459 (1963).

${ }^{5}$ C. Ren, J. D. Callen, T. A. Gianakon, C. C. Hegna, Z. Chang, E. D. Fredrickson, K. M. McGuire, G. Taylor, and M. C. Zarnstorff, Phys. Plasmas 5, 450 (1998).
${ }^{6}$ Z. Chang, J. D. Callen, E. D. Fredrickson, R. V. Budny, C. C. Hegna, K. M. McGuire, M. C. Zarnstorff, and TFTR Group, Phys. Rev. Lett. 74, 4663 (1995)

${ }^{7}$ A. Pletzer, A. Bondeson, and R. L. Dewar, J. Comput. Phys. 115, 530 (1994).

${ }^{8}$ K. Hoshino, M. Mori, T. Yamamoto, H. Tamai, T. Shoji, Y. Miura, H. Aikawa, S. Kasai, T. Kawakami, H. Kawashima, M. Maeno, T. Matsuda, K. Oasa, K. Odajima, H. Ogawa, T. Ogawa, T. Seike, T. Shiina, K. Uehara, T. Yamauchi, N. Suzuki, and H. Maeda, Phys. Rev. Lett. 69, 2208 (1992); G. Kurita, T. Tsuda, M. Azumi, T. Takizuka, and T. Takeda, Nucl. Fusion 34, 1497 (1994); C. C. Hegna and J. D. Callen, Phys. Plasmas 4, 2940 (1997).

${ }^{9}$ H. P. Furth, P. H. Rutherford, and H. Selberg, Phys. Fluids 16, 1054 (1973).

${ }^{10}$ J. Wesson, Tokamaks (Cambridge University Press, Cambridge, 1987), p. 150.

${ }^{11}$ J. L. Johnson, J. M. Greene, and B. Coppi, Phys. Fluids 6, 1169 (1963); R. D. Hazeltine and J. D. Meiss, Plasma Confinement (Addison-Wesley, New York, 1992), p. 281.

${ }^{12}$ C. C. Hegna and J. D. Callen, Phys. Plasmas 1, 2308 (1994).

${ }^{13}$ C. Mercier, Nucl. Fusion 1, 47 (1960).

${ }^{14}$ A. H. Glasser, J. M. Greene, and J. L. Johnson, Phys. Fluids 19, 567 (1976).

${ }^{15}$ R. L. Dewar and M. Persson, Phys. Fluids B 5, 4273 (1993).

${ }^{16}$ G. Schmidt, Physics of High Temperature Plasmas (Academic, New York, 1979), p. 146.

${ }^{17}$ W. A. Newcomb, Ann. Phys. (N.Y.) 10, 232 (1960).

${ }^{18}$ M. Abramowitz and I. A. Stegun, Handbook of Mathematical Functions (Dover, New York, 1970), p. 896.

${ }^{19}$ R. C. Grimm, J. M. Greene, and J. L. Johnson, Methods Comput. Phys. 9, 253 (1976).

${ }^{20}$ J. M. Greene, Comments Plasma Phys. Control. Fusion 17, 389 (1997).

${ }^{21}$ V. D. Shafranov and E. I. Yurchenko, Sov. Phys. JETP 26, 682 (1968).

${ }^{22}$ V. E. Lynch, B. A. Carreras, H. R. Hicks, J. A. Holmes, and L. Garcia, Comput. Phys. Commun. 24, 465 (1981). 